УДК 517.958

\title{
Homogenization of Acoustic Equations for a Partially Perforated Elastic Material with Slightly Viscous Fluid
}

\author{
Alexey S. Shamaev* \\ Vladlena V. Shumilova ${ }^{\dagger}$ \\ Institute for Problems in Mechanics of RAS \\ Vernadskogo, 101-1, Moscow, 119526
}

Russia

Received 15.04.2015, received in revised form 10.05.2015, accepted 25.06.2015

$\overline{\text { In this paper a mathematical model describing small oscillations of a heterogeneous medium is considered. }}$. The medium consists of a partially perforated elastic material and a slightly viscous compressible fluid filling the pores. For the given model the corresponding homogenized problem is constructed by using the two-scale convergence method. The boundary conditions connecting equations of the homogenized model on the boundary between the continuous elastic material and the porous elastic material with fluid are found.

Keywords: homogenization, two-scale convergence, heterogeneous medium.

DOI: $10.17516 / 1997-1397-2015-8-3-356-370$

In 1989, Nguetseng [1] introduced the notion of two-scale convergence, which provides a new approach in the homogenization theory. The method of two-scale convergence was further developed by Allaire [2] and generalized by other authors (see, e.g., [3-6]). As it turns out, this method is especially useful for studying homogenization problems whose solutions do not have a limit in the classical sense (for example, in the $L^{2}$-norm). In applications, such problems describe some physical processes in heterogeneous media, for example, a diffusion process in highly heterogeneous media [2] or a joint motion of an elastic skeleton and a slightly viscous fluid [7]. Recently, the method of two-scale convergence is widely applied in the homogenization of various mathematical problems that arise in mechanics of heterogeneous media (see, e.g., [8-14]).

In this paper, we consider a mathematical problem that describes small oscillations of a heterogeneous medium consisting of a partially perforated elastic material and a slightly viscous compressible fluid filling the pores. We assume that the elastic material is inhomogeneous with $\varepsilon$-periodic microstructure, and the structure of the perforation in the porous part of the elastic material is also $\varepsilon$-periodic. The mathematical problem under consideration involves the linear elasticity system describing the motion of the elastic material, and the Stokes system describing the motion of the fluid. Finally, the problem is complemented by homogeneous boundary and initial conditions. Using the method of two-scale convergence and the Laplace transforms, we construct the corresponding homogenized problem and find the boundary conditions which connect equations of the homogenized problem on the boundary between the continuous elastic material and the porous elastic material with fluid. In addition, using the notion of strong two-scale convergence, we establish some corrector-type results under suitable smoothness assumptions on the solution of the homogenized problem and on the external force. When an

\footnotetext{
*sham@rambler.ru

$\dagger$ v.v.shumilova@mail.ru

(C) Siberian Federal University. All rights reserved
} 
elastic part of the heterogeneous medium is completely perforated, the corresponding homogenization problem was analyzed in $[7,9,12]$ and [15]. Namely, the first research of this problem was carried out in [15], and later the homogenized model was mathematically rigorously justified in [7] by using the method of two-scale convergence. In [9] and [12], this homogenized problem was derived in the form that is known from the classical physical papers such as [16] and [17].

\section{Statement of the problem}

Let $\Omega$ be a bounded domain in $\mathbb{R}^{3}$ with smooth boundary $\partial \Omega$, and let $Y=(0,1)^{3}$ be the unit cube in $\mathbb{R}^{3}$. We suppose that $\Omega=\Omega_{0} \cup \Omega_{1} \cup S$ and $Y=Y^{h} \cup Y^{s} \cup \Gamma$, where $\Omega_{0}, \Omega_{1}, Y^{h}$, and $Y^{s}$ are open connected sets in $\mathbb{R}^{3}, S$ is the smooth surface that separates $\Omega_{0}$ and $\Omega_{1}$, and $\Gamma$ is the smooth surface that separates $Y^{h}$ and $Y^{s}$. In addition, we denote by $Y_{p e r}^{h}$ (respectively, $Y_{\text {per }}^{s}$ ) the $Y$-periodic repetition of the set $Y^{h} \cup\left(\partial Y^{h} \cap \partial Y\right)$ (respectively, $\left.Y^{s} \cup\left(\partial Y^{s} \cap \partial Y\right)\right)$ and suppose that both sets $Y_{p e r}^{h}$ and $Y_{\text {per }}^{s}$ are connected in $\mathbb{R}^{3}$.

For a sufficiently small $\varepsilon>0$ we divide the domain $\Omega$ into two subdomains $\Omega_{\varepsilon}^{h}$ and $\Omega_{\varepsilon}^{s}$ as follows:

$$
\Omega_{\varepsilon}^{s}=\Omega_{1} \cap \varepsilon Y_{\text {per }}^{s}, \quad \Omega_{\varepsilon}^{h}=\Omega_{0} \cup \Omega_{1 \varepsilon}^{h} \cup\left(\partial \Omega_{1 \varepsilon}^{h} \cap S\right), \quad \Omega_{1 \varepsilon}^{h}=\Omega_{1} \cap \varepsilon Y_{\text {per }}^{h} .
$$

We suppose that the set $\Omega_{\varepsilon}^{h}$ is occupied by an elastic material, whereas the set $\Omega_{\varepsilon}^{s}$ is occupied by a slightly viscous compressible fluid. In the sequel, the sets $\Omega_{\varepsilon}^{h}$ and $\Omega_{\varepsilon}^{s}$ are called the elastic and the fluid parts of $\Omega$, respectively.

Now we are going to state the mathematical problem describing the joint motion of elastic and fluid parts of $\Omega$. Let us assume that $u^{\varepsilon}(x, t)$ is the displacement vector, $e_{k h}\left(u^{\varepsilon}\right)$ are the components of the strain tensor, $e_{k h}\left(u^{\varepsilon}\right)=\left(\partial u_{k}^{\varepsilon} / \partial x_{h}+\partial u_{h}^{\varepsilon} / \partial x_{k}\right) / 2$, and $f(x, t) \in H^{2}\left(0, T ; L^{2}(\Omega)^{3}\right)$ is a given force. The equations of motion in the elastic part $\Omega_{\varepsilon}^{h}$ are given by

$$
\rho_{0}^{\varepsilon} \frac{\partial^{2} u_{i}^{\varepsilon}}{\partial t^{2}}=\frac{\partial \sigma_{i j}^{\varepsilon}}{\partial x_{j}}+f_{i}(x, t) \text { in } \Omega_{\varepsilon}^{h} \times(0, T),
$$

where $\rho_{0}^{\varepsilon}(x)$ is the density of the elastic material,

$$
\rho_{0}^{\varepsilon}(x)=\rho_{0}\left(\varepsilon^{-1} x\right), \quad \rho_{0}(y) \in L_{p e r}^{\infty}(Y), \quad 1 \leqslant \rho_{0}(y) \leqslant \rho_{1} \quad\left(\rho_{1}=\text { const }>1\right),
$$

("per" denotes $Y$-periodicity), $\sigma_{i j}^{\varepsilon}$ are the components of the stress tensor, $\sigma_{i j}^{\varepsilon}=a_{i j k h}^{\varepsilon}(x) e_{k h}\left(u^{\varepsilon}\right)$, and $a_{i j k h}^{\varepsilon}(x)=a_{i j k h}\left(\varepsilon^{-1} x\right)$ are the elasticity coefficients such that $a_{i j k h}(y) \in L_{p e r}^{\infty}(Y)$ and

$$
\begin{gathered}
a_{i j k h}(y)=a_{j i k h}(y)=a_{k h i j}(y)=a_{i j h k}(y), \quad 1 \leqslant i, j, k, h \leqslant 3, \\
a_{i j k h}(y) \xi_{i j} \xi_{k h} \geqslant c_{0} \xi_{i j} \xi_{i j}, \quad c_{0}>0, \quad \forall \xi_{i j} \in \mathbb{R}, \quad \xi_{i j}=\xi_{j i} .
\end{gathered}
$$

Here and throughout this paper, we use the convention that repeated indices imply summation from 1 to 3 .

The equations of motion in the fluid part $\Omega_{\varepsilon}^{s}$ are given by

$$
\rho^{s} \frac{\partial^{2} u_{i}^{\varepsilon}}{\partial t^{2}}=\frac{\partial \sigma_{i j}^{\varepsilon}}{\partial x_{j}}+f_{i}(x, t) \text { in } \Omega_{\varepsilon}^{s} \times(0, T),
$$

where $\rho^{s}$ is the fluid density, $\rho^{s}=$ const $>0$, and

$$
\sigma_{i j}^{\varepsilon}=-\delta_{i j} p^{\varepsilon}+\varepsilon^{2}\left(\eta \delta_{i j} \delta_{k h}+2 \mu \delta_{i k} \delta_{j h}\right) e_{k h}\left(\frac{\partial u^{\varepsilon}}{\partial t}\right), \quad p^{\varepsilon}(x, t)=-\gamma \operatorname{div} u^{\varepsilon}(x, t) .
$$


Here $p^{\varepsilon}(x, t)$ is the fluid pressure, $\delta_{i j}$ is the Kronecker symbol, $\gamma=c^{2} \rho^{s}, c$ is the speed of sound in the fluid, $c=$ const $>0$, and $\varepsilon^{2} \eta$ and $\varepsilon^{2} \mu$ are the viscosity coefficients of the fluid satisfying the following conditions: $\mu>0$ and $\eta / \mu>-(2 / 3) \alpha$ with $0<\alpha<1$ [15].

Besides, at the interface $S_{\varepsilon}=\partial \Omega_{\varepsilon}^{h} \cap \partial \Omega_{\varepsilon}^{s}$ we have the continuity of the displacement and of the normal stress:

$$
\left[u^{\varepsilon}\right]_{S_{\varepsilon}}=0, \quad\left[\sigma_{i j}^{\varepsilon} n_{j}\right]_{S_{\varepsilon}}=0
$$

where $[\cdot]_{S_{\varepsilon}}$ denotes the jump across the boundary $S_{\varepsilon}$, and $n_{j}, j=1,2,3$, are the components of the unit normal to $S_{\varepsilon}$.

Finally, the problem is supplemented by homogeneous initial and boundary conditions

$$
u^{\varepsilon}(x, 0)=\frac{\partial u^{\varepsilon}}{\partial t}(x, 0)=0, x \in \Omega ; \quad u^{\varepsilon}(x, t)=0, x \in \partial \Omega, \quad t \in(0, T) .
$$

The variational formulation of problem (1)-(4) is the following: find a function $u^{\varepsilon}(t)$ with values in $H_{0}^{1}(\Omega)^{3}$ such that

$$
\begin{gathered}
\int_{\Omega} \rho^{\varepsilon} \frac{\partial^{2} u_{i}^{\varepsilon}}{\partial t^{2}} v_{i} d x+\varepsilon^{2} b^{\varepsilon}\left(\frac{\partial u^{\varepsilon}}{\partial t}, v\right)+c^{\varepsilon}\left(u^{\varepsilon}, v\right)=\int_{\Omega} f_{i} v_{i} d x \quad \forall v \in H_{0}^{1}(\Omega)^{3}, \\
u^{\varepsilon}(0)=\frac{\partial u^{\varepsilon}}{\partial t}(0)=0,
\end{gathered}
$$

where $\rho^{\varepsilon}(x)=\rho_{0}^{\varepsilon}(x)$ for $x \in \Omega_{\varepsilon}^{h}, \rho^{\varepsilon}(x)=\rho^{s}$ for $x \in \Omega_{\varepsilon}^{s}$, and

$$
\begin{gathered}
b^{\varepsilon}(u, v)=\int_{\Omega_{\varepsilon}^{s}}\left(\eta \operatorname{div} u \operatorname{div} v+2 \mu e_{i j}(u) e_{i j}(v)\right) d x, \\
c^{\varepsilon}(u, v)=\int_{\Omega_{\varepsilon}^{s}} \gamma \operatorname{div} u \operatorname{div} v d x+\int_{\Omega_{\varepsilon}^{h}} a_{i j k h}^{\varepsilon} e_{k h}(u) e_{i j}(v) d x .
\end{gathered}
$$

In the same way as in [15], where the whole elastic part of $\Omega$ was supposed to be porous, one can show that for any $\varepsilon>0$ there exists a unique solution of problem (5), (6).

Let us extend the vector function $f(x, t)$ by zero for $t<0$ and $t>T$. Next, we convert the evolutionary problem (1)-(4) into the stationary one by using the Laplace transform $g(t) \rightarrow g_{\lambda}$ in time. Then the variational formulation (5), (6) becomes: for a fixed $\lambda$ with $\operatorname{Re} \lambda>\lambda_{0}>0$, find a function $u_{\lambda}^{\varepsilon}(x) \in H_{0}^{1}(\Omega)^{3}$ such that

$$
\lambda^{2} \int_{\Omega} \rho^{\varepsilon}\left(u_{\lambda}^{\varepsilon}\right)_{i} v_{i} d x+\lambda \varepsilon^{2} b^{\varepsilon}\left(u_{\lambda}^{\varepsilon}, v\right)+c^{\varepsilon}\left(u_{\lambda}^{\varepsilon}, v\right)=\int_{\Omega}\left(f_{\lambda}\right)_{i} v_{i} d x \quad \forall v \in H_{0}^{1}(\Omega)^{3}
$$

\section{Two-scale convergence}

We begin this section with two basic definitions related to the theory of two-scale convergence (see $[2,5])$.

Let $u^{\varepsilon}(x)$ be a bounded sequence in $L^{2}(\Omega)$.

Definition 1. A sequence $u^{\varepsilon}(x)$ weakly two-scale converges to a function $u(x, y) \in L^{2}(\Omega \times Y, d x \times$ $d y)=L^{2}(\Omega \times Y), u^{\varepsilon}(x) \stackrel{2}{\rightarrow} u(x, y)$, if

$$
\lim _{\varepsilon \rightarrow 0} \int_{\Omega} u^{\varepsilon}(x) \varphi(x) \psi\left(\varepsilon^{-1} x\right) d x=\int_{\Omega} \int_{Y} u(x, y) \varphi(x) \psi(y) d x d y
$$

for any functions $\varphi(x) \in C_{0}^{\infty}(\Omega)$ and $\psi(y) \in C_{\text {per }}^{\infty}(Y)$. 
It should be noted that the class of test functions used in the above definition can be enlarged. For example, one can takes in (8) test functions $\varphi(x) \in C(\bar{\Omega})$ and $\psi(y) \in L_{\text {per }}^{2}(Y)$ or $\varphi(x) \in L^{2}(\Omega)$ and $\psi(y) \in C_{\text {per }}(Y)$ (see $\left.[2,5]\right)$.

Definition 2. A sequence $u^{\varepsilon}(x) \in L^{2}(\Omega)$ strongly two-scale converges to a function $u(x, y) \in$ $L^{2}(\Omega \times Y), u^{\varepsilon}(x) \stackrel{2}{\rightarrow} u(x, y)$, if

$$
\lim _{\varepsilon \rightarrow 0} \int_{\Omega} u^{\varepsilon}(x) v^{\varepsilon}(x) d x=\int_{\Omega} \int_{Y} u(x, y) v(x, y) d x d y \quad \text { whenever } \quad v^{\varepsilon}(x) \stackrel{2}{\rightarrow} v(x, y) .
$$

Let us briefly recall the main properties of two-scale convergence, the proofs of which can be found in $[2,4]$ and $[5]$.

(i) If $u^{\varepsilon}(x)$ is a bounded sequence in $L^{2}(\Omega)$, then there exists a function $u(x, y) \in L^{2}(\Omega \times Y)$ such that, up to a subsequence, $u^{\varepsilon}(x) \stackrel{2}{\rightarrow} u(x, y)$.

(ii) If $u^{\varepsilon}(x) \stackrel{2}{\rightarrow} u(x, y)$, then

$$
u^{\varepsilon}(x) \rightarrow \int_{Y} u(x, y) d y \quad \text { in } \quad L^{2}(\Omega), \quad \liminf _{\varepsilon \rightarrow 0}\left\|u^{\varepsilon}(x)\right\|_{L^{2}(\Omega)} \geqslant\|u(x, y)\|_{L^{2}(\Omega \times Y)} .
$$

(iii) If $a(y) \in L_{\text {per }}^{\infty}(Y)$ and $u^{\varepsilon}(x) \stackrel{2}{\rightarrow} u(x, y)$, then $a\left(\varepsilon^{-1} x\right) u^{\varepsilon}(x) \stackrel{2}{\rightarrow} a(y) u(x, y)$.

(iv) If $u^{\varepsilon}(x) \stackrel{2}{\rightarrow} u(x, y)$ and $\lim _{\varepsilon \rightarrow 0}\left\|u^{\varepsilon}(x)\right\|_{L^{2}(\Omega)}=\|u(x, y)\|_{L^{2}(\Omega \times Y)}$, then $u^{\varepsilon}(x) \stackrel{2}{\rightarrow} u(x, y)$.

(v) If $u^{\varepsilon}(x) \stackrel{2}{\rightarrow} u(x, y)$ and $u(x, y) \in C\left(\bar{\Omega}, L_{p e r}^{2}(Y)\right)$, then

$$
\lim _{\varepsilon \rightarrow 0}\left\|u^{\varepsilon}(x)-u\left(x, \varepsilon^{-1} x\right)\right\|_{L^{2}(\Omega)}=0 .
$$

(vi) Let $u^{\varepsilon}(x)$ be a bounded sequence in $H^{1}(\Omega)$. Then there exist functions $u(x) \in H^{1}(\Omega)$ and $u_{1}(x, y) \in L^{2}\left(\Omega, H_{p e r}^{1}(Y) / \mathbb{R}\right)$ such that, up to a subsequence, $u^{\varepsilon}(x) \stackrel{2}{\rightarrow} u(x)$ and $\nabla u^{\varepsilon}(x) \stackrel{2}{\rightarrow} \nabla u(x)+\nabla_{y} u_{1}(x, y)$. Moreover, $u(x) \in H_{0}^{1}(\Omega)$ if $u^{\varepsilon}(x) \in H_{0}^{1}(\Omega)$.

(vii) Let $u^{\varepsilon}(x)$ be a sequence in $H^{1}\left(\Omega_{1}\right)$ such that

$$
\left\|u^{\varepsilon}\right\|_{L^{2}\left(\Omega_{1}\right)} \leqslant C, \quad\left\|\nabla u^{\varepsilon}\right\|_{L^{2}\left(\Omega_{1 \varepsilon}^{h}\right)^{3}} \leqslant C, \quad \varepsilon\left\|\nabla u^{\varepsilon}\right\|_{L^{2}\left(\Omega_{\varepsilon}^{s}\right)^{3}} \leqslant C
$$

where $C$ is a positive constant which does not depend on $\varepsilon$. Then there exist functions $u(x) \in H^{1}\left(\Omega_{1}\right), u_{1}(x, y) \in L^{2}\left(\Omega_{1}, H_{p e r}^{1}\left(Y^{h}\right) / \mathbb{R}\right)$, and $w(x, y) \in L^{2}\left(\Omega_{1}, H_{p e r}^{1}(Y)\right)$ with $w(x, y)=0$ for $y \in \overline{Y^{h}}$, such that, up to a subsequence,

$$
\begin{gathered}
u^{\varepsilon}(x) \stackrel{2}{\rightarrow} u(x)+w(x, y), \quad \chi\left(\Omega_{1 \varepsilon}^{h}\right) \nabla u^{\varepsilon}(x) \stackrel{2}{\rightarrow} \chi\left(Y^{h}\right)\left(\nabla u(x)+\nabla_{y} u_{1}(x, y)\right) . \\
\varepsilon \chi\left(\Omega_{\varepsilon}^{s}\right) \nabla u^{\varepsilon}(x) \stackrel{2}{\rightarrow} \chi\left(Y^{s}\right) \nabla_{y} w(x, y),
\end{gathered}
$$

where $\chi(D)$ denotes the characteristic function of the set $D$. Moreover, $u(x) \in H_{0}^{1}(\Omega)$ if $u^{\varepsilon}(x) \in H_{0}^{1}(\Omega)$. 
Now, using the above properties of two-scale convergence, we are going to study the asymptotic behavior of the solution $u_{\lambda}^{\varepsilon}$ of problem (7) when $\varepsilon$ goes to 0 .

Firstly, choosing in (7) $v=u_{\lambda}^{\varepsilon}$, we obtain

$$
c^{\varepsilon}\left(u_{\lambda}^{\varepsilon}, u_{\lambda}^{\varepsilon}\right) \leqslant C, \quad \varepsilon^{2} b^{\varepsilon}\left(u_{\lambda}^{\varepsilon}, u_{\lambda}^{\varepsilon}\right) \leqslant C .
$$

Hereinafter, $C$ denotes various positive constants independent of $\varepsilon$.

By using the same arguments as in [7], we deduce that the solution $u_{\lambda}^{\varepsilon}$ of problem (7) satisfies the a priory estimates

$$
\left\|u_{\lambda}^{\varepsilon}\right\|_{L^{2}(\Omega)^{3}} \leqslant C, \quad \varepsilon\left\|\nabla\left(u_{\lambda}^{\varepsilon}\right)_{i}\right\|_{L^{2}\left(\Omega_{\varepsilon}^{s}\right)^{3}} \leqslant C, \quad\left\|\nabla\left(u_{\lambda}^{\varepsilon}\right)_{i}\right\|_{L^{2}\left(\Omega_{\varepsilon}^{h}\right)^{3}} \leqslant C, \quad\left\|\operatorname{div} u_{\lambda}^{\varepsilon}\right\|_{L^{2}\left(\Omega_{1}\right)} \leqslant C .
$$

For notational convenience we denote by $\nabla u_{\lambda}^{\varepsilon}$ the $3 \times 3$ matrix with coefficients $\partial\left(u_{\lambda}^{\varepsilon}\right)_{i} / \partial x_{j}$. In order to proceed we need the following crucial lemma.

Lemma 1. Let $u_{\lambda}^{\varepsilon}$ be a solution of problem (7). Then, up to a subsequence,

$$
\begin{gathered}
u_{\lambda}^{\varepsilon}(x) \stackrel{2}{\rightarrow} u_{\lambda}(x) \quad \text { for } \quad x \in \Omega_{0}, \quad u_{\lambda}^{\varepsilon}(x) \stackrel{2}{\rightarrow} u_{\lambda}(x)+w_{\lambda}(x, y) \text { for } x \in \Omega_{1}, \\
\nabla u_{\lambda}^{\varepsilon}(x) \stackrel{2}{\rightarrow} \nabla u_{\lambda}(x)+\nabla_{y} u_{\lambda}^{0}(x, y) \text { for } x \in \Omega_{0}, \\
\chi\left(\Omega_{1 \varepsilon}^{h}\right) \nabla u_{\lambda}^{\varepsilon}(x) \stackrel{2}{\rightarrow} \chi\left(Y^{h}\right)\left(\nabla u_{\lambda}(x)+\nabla_{y} u_{\lambda}^{1}(x, y)\right) \text { for } x \in \Omega_{1}, \\
\varepsilon \chi\left(\Omega_{\varepsilon}^{s}\right) \nabla u_{\lambda}^{\varepsilon}(x) \stackrel{2}{\rightarrow} \chi\left(Y^{s}\right) \nabla_{y} w_{\lambda}(x, y) \text { for } x \in \Omega_{1},
\end{gathered}
$$

where

$$
\begin{gathered}
u_{\lambda} \in H_{0}^{1}(\Omega)^{3}, \quad u_{\lambda}^{0} \in L^{2}\left(\Omega_{0}, H_{p e r}^{1}(Y)^{3} / \mathbb{R}^{3}\right), \quad u_{\lambda}^{1} \in L^{2}\left(\Omega_{1}, H_{p e r}^{1}\left(Y^{h}\right)^{3} / \mathbb{R}^{3}\right), \\
w_{\lambda} \in L^{2}\left(\Omega_{1}, H_{p e r}^{1}(Y)^{3}\right), \quad w_{\lambda}=0 \text { for } y \in \overline{Y^{h}}, \quad \operatorname{div}_{y} w_{\lambda}=0 \text { for } y \in Y^{s} .
\end{gathered}
$$

Proof. Using the above estimates and properties (vi) and (vii) of two-scale convergence, we have, up to a subsequence,

$$
u_{\lambda}^{\varepsilon}(x) \stackrel{2}{\rightarrow} v_{\lambda}^{0}(x) \text { for } x \in \Omega_{0}, \quad u_{\lambda}^{\varepsilon}(x) \stackrel{2}{\rightarrow} v_{\lambda}^{1}(x)+w_{\lambda}(x, y) \text { for } x \in \Omega_{1},
$$

where $v_{\lambda}^{0} \in H^{1}\left(\Omega_{0}\right)^{3}, v_{\lambda}^{1} \in H^{1}\left(\Omega_{1}\right)^{3}$, and $w_{\lambda} \in L^{2}\left(\Omega_{1}, H_{\text {per }}^{1}(Y)^{3}\right)$ with $w_{\lambda}=0$ for $y \in \overline{Y^{h}}$. Besides, in virtue of property (vii), we also derive relation (13). Furthermore, relation (13) and the last estimate in (9) yield $\operatorname{div}_{y} w_{\lambda}=0$ for $y \in Y^{s}$.

To prove that $\left.v_{\lambda}^{0}\right|_{S}=\left.v_{\lambda}^{1}\right|_{S}$, we extend the perforation from $\Omega_{1}$ to $\Omega_{0}$ by setting $\Omega_{\varepsilon}=\Omega \cap \varepsilon Y_{p e r}^{h}$. Then, up to a subsequence,

$$
\chi\left(\Omega_{\varepsilon}\right) u_{\lambda}^{\varepsilon}(x) \rightarrow\left|Y^{h}\right| u_{\lambda}(x) \text { in } L^{2}(\Omega)^{3}
$$

(see [18]), where $u_{\lambda} \in H_{0}^{1}(\Omega)^{3}$. On the other hand, from (14) we obtain

$$
\chi\left(\Omega_{\varepsilon} \cap \Omega_{0}\right) u_{\lambda}^{\varepsilon}(x) \rightarrow\left|Y^{h}\right| v_{\lambda}^{0}(x) \text { in } L^{2}\left(\Omega_{0}\right)^{3}, \quad \chi\left(\Omega_{1 \varepsilon}^{h}\right) u_{\lambda}^{\varepsilon}(x) \rightarrow\left|Y^{h}\right| v_{\lambda}^{1}(x) \text { in } L^{2}\left(\Omega_{1}\right)^{3} .
$$

It is easy to see that $u_{\lambda}(x)=v_{\lambda}^{0}(x)$ if $x \in \Omega_{0}$ and $u_{\lambda}(x)=v_{\lambda}^{1}(x)$ if $x \in \Omega_{1}$, therefore $\left.v_{\lambda}^{0}\right|_{S}=\left.v_{\lambda}^{1}\right|_{S}$. Finally, relations (11) and (12) immediately follow from property (vi) of two-scale convergence. 


\section{Limiting behavior of the pressure}

In this section we study the limit behavior of the fluid pressure $p_{\lambda}^{\varepsilon}(x)$. Namely, we state and prove the following lemma.

Lemma 2. Let $u_{\lambda}^{\varepsilon}$ be a solution of problem (7), and let $p_{\lambda}^{\varepsilon}=-\gamma \operatorname{div} u_{\lambda}^{\varepsilon}$ in $\Omega_{\varepsilon}^{s}$. Then, up to a subsequence,

$$
\chi\left(\Omega_{\varepsilon}^{s}\right) p_{\lambda}^{\varepsilon}(x) \stackrel{2}{\rightarrow} \chi\left(Y^{s}\right) p_{\lambda}(x), \quad p_{\lambda}(x) \in L^{2}\left(\Omega_{1}\right) .
$$

Moreover,

$$
\int_{Y^{h}} \operatorname{div}_{y} u_{\lambda}^{1}(x, y) d y=\left|Y^{s}\right| \operatorname{div} u_{\lambda}(x)+\frac{\left|Y^{s}\right|}{\gamma} p_{\lambda}(x)+\operatorname{div} \int_{Y^{s}} w_{\lambda}(x, y) d y .
$$

Proof. From (9) it follows that the sequence $\chi\left(\Omega_{\varepsilon}^{s}\right) p_{\lambda}^{\varepsilon}$ is bounded in $L^{2}\left(\Omega_{1}\right)$. Therefore, up to a subsequence, we can assume $\chi\left(\Omega_{\varepsilon}^{s}\right) p_{\lambda}^{\varepsilon}(x) \stackrel{2}{\rightarrow} p_{\lambda}(x, y)$ for $x \in \Omega_{1}$.

Now we take a test vector function of the form $v(x)=\varepsilon \varphi(x) b\left(\varepsilon^{-1} x\right)$, where $\varphi(x) \in C_{0}^{\infty}\left(\Omega_{1}\right)$, $b(y) \in H_{p e r}^{1}(Y)^{3}$, supp $b(y) \subset Y^{s}$. Passing to the two-scale limit in the integral identity (7) as $\varepsilon \rightarrow 0$, we have

$$
\int_{\Omega_{1}} \int_{Y^{s}} p_{\lambda}(x, y) \varphi(x) \operatorname{div}_{y} b(y) d x d y=0
$$

or, since $\varphi(x)$ is arbitrary,

$$
\int_{Y^{s}} p_{\lambda}(x, y) \operatorname{div}_{y} b(y) d y=0
$$

which implies $p_{\lambda}(x, y)=p_{\lambda}(x)$ for $y \in Y^{s}$. Relation (15) follows now from property (iii) of two-scale convergence.

To prove equality (16), we use Lemma 1 and get

$$
\begin{gathered}
\operatorname{div} u_{\lambda}^{\varepsilon} \rightarrow \operatorname{div} u_{\lambda}(x)+\operatorname{div} \int_{Y^{s}} w_{\lambda}(x, y) d y \text { in } L^{2}\left(\Omega_{1}\right), \\
\chi\left(\Omega_{1 \varepsilon}^{h}\right) \operatorname{div} u_{\lambda}^{\varepsilon} \rightarrow\left|Y^{h}\right| \operatorname{div} u_{\lambda}(x)+\int_{Y^{h}} \operatorname{div}_{y} u_{\lambda}^{1}(x, y) d y \text { in } L^{2}\left(\Omega_{1}\right) .
\end{gathered}
$$

On the other hand, we already have proved that

$$
\chi\left(\Omega_{\varepsilon}^{s}\right) \operatorname{div} u_{\lambda}^{\varepsilon} \rightarrow-\frac{\left|Y^{s}\right|}{\gamma} p_{\lambda}(x) \text { in } L^{2}\left(\Omega_{1}\right) .
$$

Thus,

$$
\operatorname{div} u_{\lambda}^{\varepsilon} \rightarrow\left|Y^{h}\right| \operatorname{div} u_{\lambda}(x)-\frac{\left|Y^{s}\right|}{\gamma} p_{\lambda}(x)+\int_{Y^{h}} \operatorname{div}_{y} u_{\lambda}^{1}(x, y) d y \text { in } L^{2}\left(\Omega_{1}\right) .
$$

Comparing the last relation with (17) yields the desired equality (16).

\section{The cell Stokes problem}

Now we choose in (7) a test vector function of the form $v(x)=\xi(x) b\left(\varepsilon^{-1} x\right)$, where $\xi(x) \in$ $C_{0}^{\infty}\left(\Omega_{1}\right)$ and $b(y) \in H_{\text {per }}^{1}(Y)^{3}$ with supp $b(y) \subset Y^{s}$ and $\operatorname{div}_{y} b(y)=0$. Passing in (7) to the limit as $\varepsilon \rightarrow 0$, using Lemma 1 , and taking into account that $\xi(x)$ is arbitrary, we obtain

$$
\int_{Y^{s}}\left(\lambda^{2} \rho^{s} w_{\lambda}(x, y)-\lambda \mu \Delta_{y y} w_{\lambda}(x, y)-g_{\lambda}(x)\right) b(y) d y=0,
$$


where we denote $g_{\lambda}(x)=f_{\lambda}(x)-\lambda^{2} \rho^{s} u_{\lambda}(x)-\nabla p_{\lambda}(x)$. Since the orthogonal of divergencefree functions is exactly the gradients, from (18) it follows that there exists a function $\Phi_{\lambda} \in$ $L^{2}\left(\Omega_{1}, H_{\text {per }}^{1}\left(Y^{s}\right)\right)$ such that

$$
\lambda^{2} \rho^{s} w_{\lambda}(x, y)-\lambda \mu \Delta_{y y} w_{\lambda}(x, y)-g_{\lambda}(x)=\nabla_{y} \Phi_{\lambda}(x, y), \quad x \in \Omega_{1}, \quad y \in Y^{s} .
$$

Now, as in [12], we look for a vector function $w_{\lambda}(x, y)$ in the form

$$
w_{\lambda}(x, y)=M_{\lambda}^{r}(x) N_{\lambda}^{r}(y), \quad x \in \Omega_{1}, y \in Y^{s},
$$

where $M_{\lambda}^{r} \in L^{2}\left(\Omega_{1}\right)$ and $N_{\lambda}^{r} \in H_{p e r}^{1}(Y)^{3}, r=1,2,3$, are to be specified. To do this, we substitute (20) into (19) and get

$$
M_{\lambda}^{r}(x)\left(\lambda^{2} \rho^{s} N_{\lambda}^{r}(y)-\lambda \mu \Delta_{y y} N_{\lambda}^{r}(y)\right)-g_{\lambda}(x)=\nabla_{y} \Phi_{\lambda}(x, y) .
$$

Now we set

$$
M_{\lambda}^{r}(x)=\left(g_{\lambda}\right)_{r}(x), \quad r=1,2,3 ; \quad \Phi_{\lambda}(x, y)=-\left(g_{\lambda}\right)_{r}(x) W_{\lambda}^{r}(y) .
$$

Then from (21) it follows that

$$
\left(g_{\lambda}\right)_{r}(x)\left(\nabla_{y} W_{\lambda}^{r}(y)+\lambda^{2} \rho^{s} N_{\lambda}^{r}(y)-\lambda \mu \Delta_{y y} N_{\lambda}^{r}(y)\right)=\left(g_{\lambda}\right)_{r}(x) e^{r},
$$

where $e^{r}$ is the unit vector of the $y_{r}$-axis. Finally, we define the pair $\left\{N_{\lambda}^{r}(y), W_{\lambda}^{r}(y)\right\}$ as the solution of the following Stokes problem:

$$
\nabla_{y} W_{\lambda}^{r}+\lambda^{2} \rho^{s} N_{\lambda}^{r}-\lambda \mu \Delta_{y y} N_{\lambda}^{r}=e^{r}, \quad \operatorname{div}_{y} N_{\lambda}^{r}=0 \text { in } Y^{s}, N_{\lambda}^{r}=0 \text { on } \Gamma .
$$

Let us summarize the results of this section in the following lemma.

Lemma 3. Let $w_{\lambda}(x, y)$ be as in Lemma 1. Then

$$
w_{\lambda}(x, y)=\left(\left(f_{\lambda}\right)_{r}(x)-\lambda^{2} \rho^{s}\left(u_{\lambda}\right)_{r}(x)-\frac{\partial p_{\lambda}}{\partial x_{r}}(x)\right) N_{\lambda}^{r}(y),
$$

where $N_{\lambda}^{r}(y), r=1,2,3$, are the solutions of the cell Stokes problems (22).

\section{Homogenized tensors}

Lemma 4. Let $u_{\lambda}^{\varepsilon}$ be a solution of problem (7). Then

$$
\begin{gathered}
a_{i j k h}^{\varepsilon} e_{k h}\left(u_{\lambda}^{\varepsilon}\right) \rightarrow b_{i j k h} e_{k h}\left(u_{\lambda}\right) \text { in } L^{2}\left(\Omega_{0}\right), \\
\chi\left(\Omega_{1 \varepsilon}^{h}\right) a_{i j k h}^{\varepsilon} e_{k h}\left(u_{\lambda}^{\varepsilon}\right) \rightarrow q_{i j k h} e_{k h}\left(u_{\lambda}\right)+\beta_{i j} p_{\lambda} \text { in } L^{2}\left(\Omega_{1}\right),
\end{gathered}
$$

where

$$
\begin{gathered}
b_{i j k h}=\int_{Y}\left(a_{i j k h}+a_{i j l m} e_{l m}^{y}\left(V^{k h}\right)\right) d y, \quad q_{i j k h}=\int_{Y^{h}}\left(a_{i j k h}-a_{i j l m} e_{l m}^{y}\left(Q^{k h}\right)\right) d y, \\
\beta_{i j}=-\int_{Y^{h}} \operatorname{div}_{y} Q^{i j}(y) d y .
\end{gathered}
$$

Here $V^{k h}(y) \in H_{\text {per }}^{1}(Y)^{3} / \mathbb{R}^{3}$ and $Q^{k h}(y) \in H_{\text {per }}^{1}\left(Y^{h}\right)^{3} / \mathbb{R}^{3}$ are the solutions of the following cell problems:

$$
\frac{\partial}{\partial y_{j}}\left(a_{i j k h}+a_{i j l m} e_{l m}^{y}\left(V^{k h}\right)\right)=0 \text { in } Y
$$




$$
\left\{\begin{array}{l}
\frac{\partial}{\partial y_{j}}\left(a_{i j k h}-a_{i j l m} e_{l m}^{y}\left(Q^{k h}\right)\right)=0 \text { in } Y^{h}, \\
\left(a_{i j k h}-a_{i j l m} e_{l m}^{y}\left(Q^{k h}\right)\right) \nu_{j}=0 \text { on } \Gamma,
\end{array}\right.
$$

where $\nu_{j}, j=1,2,3$, are the components of the unit normal to the boundary $\Gamma$.

Proof. Using the properties of two-scale convergence, we have

$$
\begin{gathered}
a_{i j k h}^{\varepsilon} e_{k h}\left(u_{\lambda}^{\varepsilon}\right) \rightarrow \int_{Y} a_{i j k h}(y)\left(e_{k h}\left(u_{\lambda}\right)+e_{k h}^{y}\left(u_{\lambda}^{0}\right)\right) d y \text { in } L^{2}\left(\Omega_{0}\right), \\
\chi\left(\Omega_{1 \varepsilon}^{h}\right) a_{i j k h}^{\varepsilon} e_{k h}\left(u_{\lambda}^{\varepsilon}\right) \rightarrow \int_{Y^{h}} a_{i j k h}(y)\left(e_{k h}\left(u_{\lambda}\right)+e_{k h}^{y}\left(u_{\lambda}^{1}\right)\right) d y \text { in } L^{2}\left(\Omega_{1}\right) .
\end{gathered}
$$

To prove (24), we take in (7) a test vector function $v(x)=\varepsilon \varphi(x) b\left(\varepsilon^{-1} x\right)$, where $\varphi(x) \in$ $C_{0}^{\infty}\left(\Omega_{0}\right), b(y) \in C_{p e r}^{\infty}(Y)^{3}$. Passing to the limit as $\varepsilon \rightarrow 0$ and using Lemma 1, we obtain

$$
\int_{Y} a_{i j k h}\left(e_{k h}\left(u_{\lambda}\right)+e_{k h}^{y}\left(u_{\lambda}^{0}\right)\right) e_{i j}^{y}(b) d y=0 .
$$

We look for a solution of (31) in the form

$$
u_{\lambda}^{0}(x, y)=V^{k h}(y) \frac{\partial\left(u_{\lambda}\right)_{k}}{\partial x_{h}}(x), \quad x \in \Omega_{0}, \quad y \in Y,
$$

where $V^{k h}(y) \in H_{\text {per }}^{1}(Y)^{3} / \mathbb{R}^{3}$. Substituting (32) into (31) yields

$$
\int_{Y}\left(a_{i j k h}+a_{i j l m} e_{l m}^{y}\left(V^{k h}\right)\right) e_{i j}^{y}(b) d y=0 .
$$

An integration by parts shows that (33) is a variational formulation associated to (27). Furthermore, we have

$$
\int_{Y} a_{i j k h}\left(e_{k h}\left(u_{\lambda}\right)+e_{k h}^{y}\left(u_{\lambda}^{0}\right)\right) d y=b_{i j k h} e_{k h}\left(u_{\lambda}\right)
$$

Comparing the last equality with (29), we derive (24).

It remains to prove (25). For this purpose we choose in $(7)$ a test vector function $v(x)=$ $\varepsilon \varphi(x) b\left(\varepsilon^{-1} x\right)$, where $\varphi(x) \in C_{0}^{\infty}\left(\Omega_{1}\right), b(y) \in C_{p e r}^{\infty}(Y)^{3}$. Passing to the limit as $\varepsilon \rightarrow 0$ and using Lemmas 1 and 2, we get

$$
\int_{Y^{h}} a_{i j k h}\left(e_{k h}\left(u_{\lambda}\right)+e_{k h}^{y}\left(u_{\lambda}^{1}\right)\right) e_{i j}^{y}(b) d y-p_{\lambda} \int_{Y^{s}} \operatorname{div}_{y} b d y=0 .
$$

We look for a solution of (34) in the form

$$
u_{\lambda}^{1}(x, y)=-Q^{k h}(y) \frac{\partial\left(u_{\lambda}\right)_{k}}{\partial x_{h}}(x)-p_{\lambda}(x) Q(y), \quad x \in \Omega_{1}, \quad y \in Y^{h},
$$

where $Q^{k h}(y), Q(y) \in H_{\text {per }}^{1}\left(Y^{h}\right)^{3} / \mathbb{R}^{3}$. Substituting (35) into (34), we obtain two integral identities:

$$
\begin{gathered}
\int_{Y^{h}}\left(a_{i j k h}-a_{i j l m} e_{l m}^{y}\left(Q^{k h}\right)\right) e_{i j}^{y}(b) d y=0, \\
\int_{Y^{h}} a_{i j k h} e_{k h}^{y}(Q) e_{i j}^{y}(b) d y-\int_{Y^{h}} \operatorname{div}_{y} b d y=0 .
\end{gathered}
$$


An integration by parts shows that (36) is a variational formulation associated to (28), while (37) is a variational formulation of the following cell problem:

$$
\frac{\partial}{\partial y_{j}}\left(a_{i j k h} e_{k h}^{y}(Q)\right)=0 \text { in } Y^{h} ; \quad a_{i j k h} e_{k h}^{y}(Q) \nu_{j}=\nu_{i} \text { on } \Gamma \text {. }
$$

For further needs, we extend $Q^{k h}(y)$ and $Q(y)$ from $Y^{h}$ to the entire periodicity cell $Y$ in such a way that the extended vector functions $\tilde{Q}^{k h}(y)$ and $\tilde{Q}(y)$ belong to $H_{p e r}^{1}(Y)^{3}$ and

$$
\left\|\tilde{Q}^{k h}\right\|_{H_{p e r}^{1}(Y)^{3}} \leqslant C\left\|Q^{k h}\right\|_{H_{p e r}^{1}\left(Y^{h}\right)^{3}}, \quad\|\tilde{Q}\|_{H_{p e r}^{1}(Y)^{3}} \leqslant C\|Q\|_{H_{p e r}^{1}\left(Y^{h}\right)^{3}} .
$$

Setting $b=\tilde{Q}$ in $(36)$ and $b=\tilde{Q}^{k h}$ in $(37)$ yields

$$
\int_{Y^{h}} a_{i j k h} e_{k h}^{y}(Q) d y=\int_{Y^{h}} \operatorname{div}_{y} Q^{i j} d y=-\beta_{i j} .
$$

Thus, in view of $(26)$ we have

$$
\int_{Y^{h}} a_{i j k h}\left(e_{k h}\left(u_{\lambda}\right)+e_{k h}^{y}\left(u_{\lambda}^{1}\right)\right) d y=q_{i j k h} e_{k h}\left(u_{\lambda}\right)+\beta_{i j} p_{\lambda} .
$$

Finally, comparing the last equality with (30), we obtain (25).

It should be noted that the homogenized coefficients $b_{i j k h}$ and $q_{i j k h}$ are real and they possess the classical properties of symmetry and ellipticity (see [7] and [19]).

To conclude this section, we substitute representation (35) into equality (16). Then

$$
\left(\frac{\Pi}{\gamma}+\beta\right) p_{\lambda}+\operatorname{div} w_{\lambda}^{0}+\alpha_{k h} e_{k h}\left(u_{\lambda}\right)=0, x \in \Omega_{1},
$$

where $\Pi=\left|Y^{s}\right|$ is the porosity, $\alpha_{i j}=\Pi \delta_{i j}-\beta_{i j}$,

$$
\beta=\int_{Y^{h}} \operatorname{div}_{y} Q(y) d y, \quad w_{\lambda}^{0}(x)=\int_{Y^{s}} w_{\lambda}(x, y) d y
$$

Note that the vector function $w_{\lambda}^{0}$ satisfies the boundary condition $w_{\lambda}^{0} \cdot \zeta=0$ on $\partial \Omega_{1}$, where $\zeta$ is the unit normal to $\partial \Omega_{1}$. Indeed, using (10), we can easily deduce that

$$
\lim _{\varepsilon \rightarrow 0} \int_{\Omega} \operatorname{div} u_{\lambda}^{\varepsilon} d x=\int_{\Omega} \operatorname{div} u_{\lambda} d x+\int_{\Omega_{1}} \operatorname{div} w_{\lambda}^{0} d x
$$

and the desired boundary condition follows immediately by integration by parts.

\section{Homogenized problem}

Now we choose in $(7)$ a test vector function $v \in H_{0}^{1}(\Omega)^{3}$, which does not depend on $\varepsilon$. Then passing in $(7)$ to the limit as $\varepsilon \rightarrow 0$ and using Lemmas 1,2 , and 4 , we obtain

$$
\begin{gathered}
\lambda^{2} \tilde{\rho}_{0} \int_{\Omega_{0}}\left(u_{\lambda}\right)_{i} v_{i} d x+\lambda^{2} \int_{\Omega_{1}}\left(\tilde{\rho}_{1}\left(u_{\lambda}\right)_{i}+\rho^{s}\left(w_{\lambda}^{0}\right)_{i}\right) v_{i} d x-\Pi \int_{\Omega_{1}} p_{\lambda} \operatorname{div} v d x+ \\
+\int_{\Omega_{0}} b_{i j k h} e_{k h}\left(u_{\lambda}\right) e_{i j}(v) d x+\int_{\Omega_{1}}\left(q_{i j k h} e_{k h}\left(u_{\lambda}\right)+\beta_{i j} p_{\lambda}\right) e_{i j}(v) d x=\int_{\Omega}\left(f_{\lambda}\right)_{i} v_{i} d x
\end{gathered}
$$


where

$$
\tilde{\rho}_{0}=\int_{Y} \rho_{0}(y) d y, \quad \tilde{\rho}_{1}=\Pi \rho^{s}+\rho_{0}^{h}, \quad \rho_{0}^{h}=\int_{Y^{h}} \rho_{0}(y) d y .
$$

Integrating by parts in (40) and using the results of Sections 4 and 5 , we conclude that the differential form of the homogenized problem corresponding to problem (7) is

$$
\begin{gathered}
\lambda^{2} \tilde{\rho}_{0}\left(u_{\lambda}\right)_{i}=\frac{\partial \sigma_{i j}^{\lambda}}{\partial x_{j}}+\left(f_{\lambda}\right)_{i} \text { in } \Omega_{0}, \\
\lambda^{2} \tilde{\rho}_{1}\left(u_{\lambda}\right)_{i}+\lambda^{2} \rho^{s}\left(w_{\lambda}^{0}\right)_{i}=\frac{\partial \sigma_{i j}^{\lambda}}{\partial x_{j}}+\left(f_{\lambda}\right)_{i} \text { in } \Omega_{1}, \\
\left(\frac{\Pi}{\gamma}+\beta\right) p_{\lambda}+\operatorname{div} w_{\lambda}^{0}+\alpha_{k h} e_{k h}\left(u_{\lambda}\right)=0 \text { in } \Omega_{1}, \\
w_{\lambda}^{0}=\left(\left(f_{\lambda}\right)_{r}-\lambda^{2} \rho^{s}\left(u_{\lambda}\right)_{r}-\frac{\partial p_{\lambda}}{\partial x_{r}}\right) \int_{Y^{s}} N_{\lambda}^{r} d y, \\
w_{\lambda}^{0} \cdot \zeta=0 \text { on } \partial \Omega_{1}, \quad u_{\lambda}=0 \text { on } \partial \Omega, \quad\left[u_{\lambda}\right]_{S}=0, \quad\left[\sigma_{i j}^{\lambda} n_{j}\right]_{S}=0,
\end{gathered}
$$

where $n_{j}$ are the components of the unit normal to $S, \sigma_{i j}^{\lambda}=b_{i j k h} e_{k h}\left(u_{\lambda}\right)$ in $\Omega_{0}$ and $\sigma_{i j}^{\lambda}=$ $q_{i j k h} e_{k h}\left(u_{\lambda}\right)-\alpha_{i j} p_{\lambda}$ in $\Omega_{1}$.

Using the general theory of elliptic problems (see, e.g., [20]), one can prove that there exists a unique solution of problem (41)-(45).

Now our aim is to derive the non-stationary homogenized problem in the original variables $x$ and $t$. For this purpose, we apply the inverse Laplace transform to (23) and obtain

$$
w(x, y, t)=\left(f_{r}(x, t)-\rho^{s} \frac{\partial^{2} u_{r}}{\partial t^{2}}(x, t)-\frac{\partial p}{\partial x_{r}}(x, t)\right) * N^{r}(y, t),
$$

where the symbol $*$ denotes the convolution in $t$,

$$
g_{1}(t) * g_{2}(t)=\int_{0}^{t} g_{1}(t-s) g_{2}(s) d s .
$$

Now we set

$$
L^{r}(y, t)=\frac{\partial N^{r}}{\partial t}(y, t), \quad D^{r}(t)=\int_{Y^{s}} L^{r}(y, t) d y, \quad r=1,2,3 .
$$

Since $\hat{L}^{r}=\lambda \hat{N}^{r}$, it is easy to see that $L^{r}(y, t)$ is a solution of the Stokes problem

$$
\left\{\begin{array}{l}
\rho^{s} \frac{\partial L^{r}}{\partial t}-\mu \Delta_{y y} L^{r}+\nabla_{y} W^{r}=0, \quad \operatorname{div}_{y} L^{r}=0 \text { in } Y^{s} \times(0, T), \\
L^{r}(y, 0)=\left(\rho^{s}\right)^{-1} e^{r}, \quad y \in Y^{s} ; \quad L^{r}(y, t)=0, \quad y \in \Gamma, \quad t \in(0, T) .
\end{array}\right.
$$

Further, we can rewrite (46) as

$$
w(x, y, t)=\left(f_{r}(x, t)-\rho^{s} \frac{\partial^{2} u_{r}}{\partial t^{2}}(x, t)-\frac{\partial p}{\partial x_{r}}(x, t)\right) * \int_{0}^{t} L^{r}(y, \tau) d \tau
$$

and so

$$
w^{0}(x, t)=\left(f_{r}(x, t)-\rho^{s} \frac{\partial^{2} u_{r}}{\partial t^{2}}(x, t)-\frac{\partial p}{\partial x_{r}}(x, t)\right) * \int_{0}^{t} D^{r}(\tau) d \tau
$$


It is easy to check that

$$
\frac{\partial^{2} w^{0}}{\partial t^{2}}=\left(f_{r}-\rho^{s} \frac{\partial^{2} u_{r}}{\partial t^{2}}-\frac{\partial p}{\partial x_{r}}\right) * \frac{\partial D^{r}}{\partial t}+D^{r}(0)\left(f_{r}-\rho^{s} \frac{\partial^{2} u_{r}}{\partial t^{2}}-\frac{\partial p}{\partial x_{r}}\right) .
$$

Finally, we apply the inverse Laplace transform to system (41)-(43). As a result, we deduce that the homogenized problem corresponding to the original problem (1)-(4) takes the form

$$
\begin{gathered}
\tilde{\rho}_{0} \frac{\partial^{2} u_{i}}{\partial t^{2}}=\frac{\partial}{\partial x_{j}}\left(b_{i j k h} \frac{\partial u_{k}}{\partial x_{h}}\right)+f_{i}(x, t) \text { in } \Omega_{0} \times(0, T), \\
\tilde{\rho}_{1} \frac{\partial^{2} u_{i}}{\partial t^{2}}+\rho^{s}\left(f_{r}-\rho^{s} \frac{\partial^{2} u_{r}}{\partial t^{2}}-\frac{\partial p}{\partial x_{r}}\right) * \frac{\partial D_{i}^{r}}{\partial t}+\rho^{s} D_{i}^{r}(0)\left(f_{r}-\rho^{s} \frac{\partial^{2} u_{r}}{\partial t^{2}}-\frac{\partial p}{\partial x_{r}}\right)= \\
=\frac{\partial}{\partial x_{j}}\left(q_{i j k h} \frac{\partial u_{k}}{\partial x_{h}}-\alpha_{i j} p\right)+f_{i}(x, t) \text { in } \Omega_{1} \times(0, T), \\
\left(\frac{\Pi}{\gamma}+\beta\right) p+\operatorname{div}_{x}\left(f_{r}-\rho^{s} \frac{\partial^{2} u_{r}}{\partial t^{2}}-\frac{\partial p}{\partial x_{r}}\right) * \int_{0}^{t} D^{r}(\tau) d \tau+\alpha_{i j} e_{i j}(u)=0 \text { in } \Omega_{1} \times(0, T), \\
\left(\left(f_{r}-\rho^{s} \frac{\partial^{2} u_{r}}{\partial t^{2}}-\frac{\partial p}{\partial x_{r}}\right) * \int_{0}^{t} D_{j}^{r}(\tau) d \tau\right) \zeta_{j}=0 \text { on } \partial \Omega_{1}, \quad[u]_{S}=0, \quad\left[\sigma_{i j} n_{j}\right]_{S}=0, \\
u(x, 0)=\frac{\partial u}{\partial t}(x, 0)=0, x \in \Omega ; \quad u(x, t)=0, \quad x \in \partial \Omega, t \in(0, T),
\end{gathered}
$$

where $\sigma_{i j}=b_{i j k h} e_{k h}(u)$ in $\Omega_{0}$ and $\sigma_{i j}=q_{i j k h} e_{k h}(u)-\alpha_{i j} p$ in $\Omega_{1}$.

Remark that system (48) describes the propagation of acoustic waves in the homogeneous elastic material contained in $\Omega_{0}$, while system (49), (50) corresponds to the Biot model [17] and describes the propagation of acoustic waves in the heterogeneous medium contained in $\Omega_{1}$.

Finally, we analyze the boundary conditions, which connect equations of the homogenized problem on the boundary $S$ between the continuous elastic material and the porous elastic material with fluid. From Section 5 it follows that these conditions depend on the following constants: $b_{i j k h}, q_{i j k h}, \Pi$, and $\beta_{i j}$, where $b_{i j k h}$ are the homogenized elasticity coefficients for the continuous elastic material, $q_{i j k h}$ are the homogenized elasticity coefficients for the porous elastic material without fluid, $\Pi$ is the porosity of the elastic material in $\Omega_{1}$, and $\beta_{i j}$ are the coefficients, which characterize the compressibility of the porous elastic material.

\section{Strong two-scale convergence}

Our next goal is to prove the strong two-scale convergence in (10) under the additional smoothness assumptions on the solution of the homogenized problem (41)-(45) and on the external force $f$. Namely, in this section we suppose that $f_{\lambda}(x) \in C^{1}(\bar{\Omega}), u_{\lambda}(x) \in C^{3}(\bar{\Omega})$ and $p_{\lambda}(x) \in C^{2}\left(\bar{\Omega}_{1}\right)$.

Theorem 1. Let $u_{\lambda}^{\varepsilon}$ be a solution of problem $(7)$, and let $p_{\lambda}^{\varepsilon}=-\gamma \operatorname{div} u_{\lambda}^{\varepsilon}$ in $\Omega_{\varepsilon}^{s}$. Then

$$
\begin{gathered}
\lim _{\varepsilon \rightarrow 0} \int_{\Omega_{\varepsilon}^{h}}\left|u_{\lambda}^{\varepsilon}(x)-u_{\lambda}(x)\right|^{2} d x=0, \quad \lim _{\varepsilon \rightarrow 0} \int_{\Omega_{\varepsilon}^{s}}\left|p_{\lambda}^{\varepsilon}(x)-p_{\lambda}(x)\right|^{2} d x=0 \\
\lim _{\varepsilon \rightarrow 0} \int_{\Omega_{\varepsilon}^{s}}\left|u_{\lambda}^{\varepsilon}(x)-u_{\lambda}(x)-w_{\lambda}\left(x, \varepsilon^{-1} x\right)\right|^{2} d x=0
\end{gathered}
$$




$$
\begin{gathered}
\lim _{\varepsilon \rightarrow 0} \int_{\Omega_{0}}\left|e\left(u_{\lambda}^{\varepsilon}(x)-u_{\lambda}(x)-\varepsilon u_{\lambda}^{0}\left(x, \varepsilon^{-1} x\right)\right)\right|^{2} d x=0, \\
\lim _{\varepsilon \rightarrow 0} \int_{\Omega_{1 \varepsilon}^{h}}\left|e\left(u_{\lambda}^{\varepsilon}(x)-u_{\lambda}(x)-\varepsilon u_{\lambda}^{1}\left(x, \varepsilon^{-1} x\right)\right)\right|^{2} d x=0, \\
\lim _{\varepsilon \rightarrow 0} \int_{\Omega_{\varepsilon}^{s}} \varepsilon^{2}\left|e\left(u_{\lambda}^{\varepsilon}(x)-w_{\lambda}\left(x, \varepsilon^{-1} x\right)\right)\right|^{2} d x=0 .
\end{gathered}
$$

Here the triple $\left\{u_{\lambda}(x), p_{\lambda}(x), w_{\lambda}^{0}(x)\right\}$ with $w_{\lambda}^{0}(x)=\int_{Y^{s}} w_{\lambda}(x, y) d y$ is the solution of the homogenized problem (41)-(45), and $u_{\lambda}^{0}(x, y), u_{\lambda}^{1}(x, y), w_{\lambda}(x, y)$ are given by (32), (35), (23), respectively.

Proof. In the integral identity (7), we take a test vector function $v=u_{\lambda}^{\varepsilon}$ and pass to the limit as $\varepsilon \rightarrow 0$. Then

$$
\begin{aligned}
& \lim _{\varepsilon \rightarrow 0}\left(\lambda^{2} \int_{\Omega} \rho^{\varepsilon}\left|u_{\lambda}^{\varepsilon}\right|^{2} d x+2 \lambda \mu \varepsilon^{2} \int_{\Omega_{\varepsilon}^{s}}\left|e_{i j}\left(u_{\lambda}^{\varepsilon}\right)\right|^{2} d x+\gamma \int_{\Omega_{\varepsilon}^{s}}\left(\operatorname{div} u_{\lambda}^{\varepsilon}\right)^{2} d x\right)+ \\
+ & \lim _{\varepsilon \rightarrow 0} \int_{\Omega_{\varepsilon}^{h}} a_{i j k h}^{\varepsilon} e_{k h}\left(u_{\lambda}^{\varepsilon}\right) e_{i j}\left(u_{\lambda}^{\varepsilon}\right) d x=\int_{\Omega_{0}} u_{\lambda} \cdot f_{\lambda} d x+\int_{\Omega_{1}}\left(u_{\lambda}+w_{\lambda}^{0}\right) \cdot f_{\lambda} d x .
\end{aligned}
$$

Let us introduce the vector function

$$
\psi_{\lambda}^{1 \varepsilon}(x)=\varepsilon u_{\lambda}^{0}\left(x, \varepsilon^{-1} x\right)+b_{\lambda}^{1 \varepsilon}(x) \text { for } x \in \Omega_{0} ; \quad \psi_{\lambda}^{1 \varepsilon}(x)=0 \text { for } x \notin \Omega_{0},
$$

where $b_{\lambda}^{1 \varepsilon}$ is a boundary layer function in a neighborhood of $\partial \Omega_{0}$, such that $\psi_{\lambda}^{1 \varepsilon} \in H_{0}^{1}\left(\Omega_{0}\right)^{3}$ and $\left\|b_{\lambda}^{1 \varepsilon}\right\|_{H^{1}\left(\Omega_{0}\right)^{3}} \rightarrow 0$ as $\varepsilon \rightarrow 0$ (see [18]).

Denote by $z_{\lambda}^{1}(x, y)$ the right-hand side of $(35)$, where $Q^{k h}(y)$ and $Q(y)$ are replaced by $\tilde{Q}^{k h}(y)$ and $\tilde{Q}(y)$, respectively. It is easy to check that

$$
\int_{Y^{s}} \operatorname{div}_{y} z_{\lambda}^{1} d y=-\int_{Y^{h}} \operatorname{div}_{y} u_{\lambda}^{1} d y .
$$

Then, in view of (16), we obtain that $z_{1}$ satisfies the equality

$$
\int_{Y^{s}}\left(\operatorname{div} u_{\lambda}+\operatorname{div}_{x} w_{\lambda}+\operatorname{div}_{y} z_{\lambda}^{1}\right) d y=-\frac{\Pi}{\gamma} p_{\lambda} .
$$

Let us now introduce the vector function

$$
\begin{gathered}
\psi_{\lambda}^{2 \varepsilon}(x)=w_{\lambda}\left(x, \varepsilon^{-1} x\right)+\varepsilon z_{\lambda}^{1}\left(x, \varepsilon^{-1} x\right)+b_{\lambda}^{2 \varepsilon}(x)+b_{\lambda}^{3 \varepsilon}(x) \text { for } x \in \Omega_{1}, \\
\psi_{\lambda}^{2 \varepsilon}(x)=0 \text { for } x \notin \Omega_{1},
\end{gathered}
$$

where $b_{\lambda}^{2 \varepsilon}$ and $b_{\lambda}^{3 \varepsilon}$ are boundary layer functions in a neighborhood of $\partial \Omega_{1}$ (see [18]) such that $\psi_{\lambda}^{2 \varepsilon} \in H_{0}^{1}\left(\Omega_{1}\right)^{3}$ and

$$
\left\|b^{2 \varepsilon}\right\|_{H^{1}\left(\Omega_{1}\right)^{3}} \rightarrow 0, \quad\left\|b^{3 \varepsilon}\right\|_{L^{2}\left(\Omega_{1}\right)^{3}} \rightarrow 0, \quad \varepsilon\left\|\nabla b_{i}^{3 \varepsilon}\right\|_{L^{2}\left(\Omega_{1}\right)^{3}} \rightarrow 0, \quad \operatorname{supp} b^{3 \varepsilon} \subset \Omega_{\varepsilon}^{s} .
$$

Now we denote $\psi_{\lambda}^{\varepsilon}(x)=u_{\lambda}(x)+\psi_{\lambda}^{1 \varepsilon}(x)+\psi_{\lambda}^{2 \varepsilon}(x)$. By construction, the vector function $\psi_{\lambda}^{\varepsilon}$ belongs to $H_{0}^{1}(\Omega)^{3}$. Setting $v=\psi_{\lambda}^{\varepsilon}$ in $(7)$ and then passing to the two-scale limit as $\varepsilon \rightarrow 0$, we obtain

$$
\sum_{n=1}^{6} I_{n}=\int_{\Omega_{0}} u_{\lambda} \cdot f_{\lambda} d x+\int_{\Omega_{1}}\left(u_{\lambda}+w_{\lambda}^{0}\right) \cdot f_{\lambda} d x
$$


where

$$
\begin{gathered}
I_{1}=\lambda^{2} \tilde{\rho}_{0} \int_{\Omega_{0}}\left|u_{\lambda}\right|^{2} d x, \quad I_{2}=\lambda^{2} \rho_{0}^{h} \int_{\Omega_{1}}\left|u_{\lambda}\right|^{2} d x+\lambda^{2} \rho^{s} \int_{\Omega_{1}} \int_{Y^{s}}\left|u_{\lambda}+w_{\lambda}\right|^{2} d x d y \\
I_{3}=\int_{\Omega_{0}} \int_{Y} a_{i j k h}(y)\left(e_{k h}\left(u_{\lambda}\right)+e_{k h}^{y}\left(u_{\lambda}^{0}\right)\right)\left(e_{i j}\left(u_{\lambda}\right)+e_{i j}^{y}\left(u_{\lambda}^{0}\right)\right) d x d y \\
I_{4}=\int_{\Omega_{1}} \int_{Y^{h}} a_{i j k h}(y)\left(e_{k h}\left(u_{\lambda}\right)+e_{k h}^{y}\left(u_{\lambda}^{1}\right)\right)\left(e_{i j}\left(u_{\lambda}\right)+e_{i j}^{y}\left(u_{\lambda}^{1}\right)\right) d x d y \\
I_{5}=\lambda \mu \int_{\Omega_{1}} \int_{Y}\left|\nabla_{y} w_{\lambda}\right|^{2} d x d y, \quad I_{6}=\frac{\Pi}{\gamma} \int_{\Omega_{1}} p_{\lambda}^{2} d x \quad \text { (according to (59)). }
\end{gathered}
$$

By the property of lower semicontinuity (ii), the left-hand side of (58) is greater than or equal to the left-hand side of (60). Since the right-hand sides of (58) and (60) coincide, we have

$$
\begin{gathered}
\lambda^{2} \lim _{\varepsilon \rightarrow 0} \int_{\Omega_{0}} \rho^{\varepsilon}\left|u_{\lambda}^{\varepsilon}\right|^{2} d x=I_{1}, \quad \lambda^{2} \lim _{\varepsilon \rightarrow 0} \int_{\Omega_{1}} \rho^{\varepsilon}\left|u_{\lambda}^{\varepsilon}\right|^{2} d x=I_{2}, \\
\lim _{\varepsilon \rightarrow 0} \int_{\Omega_{0}} a_{i j k h}^{\varepsilon} e_{k h}\left(u_{\lambda}^{\varepsilon}\right) e_{i j}\left(u_{\lambda}^{\varepsilon}\right) d x=I_{3}, \quad \lim _{\varepsilon \rightarrow 0} \int_{\Omega_{1 \varepsilon}^{h}} a_{i j k h}^{\varepsilon} e_{k h}\left(u_{\lambda}^{\varepsilon}\right) e_{i j}\left(u_{\lambda}^{\varepsilon}\right) d x=I_{4}, \\
2 \lambda \mu \lim _{\varepsilon \rightarrow 0} \int_{\Omega_{\varepsilon}^{s}} \varepsilon^{2}\left|e_{i j}\left(u_{\lambda}^{\varepsilon}\right)\right|^{2} d x=I_{5}, \quad \gamma \lim _{\varepsilon \rightarrow 0} \int_{\Omega_{\varepsilon}^{s}}\left(\operatorname{div} u_{\lambda}^{\varepsilon}\right)^{2} d x=I_{6} .
\end{gathered}
$$

Now, using properties (iii)-(v) of two-scale convergence leads to (53) and (54). Moreover, we have

$$
\begin{gathered}
e\left(u_{\lambda}^{\varepsilon}(x)\right) \stackrel{2}{\rightarrow} e\left(u_{\lambda}(x)\right)+e_{y}\left(u_{\lambda}^{0}(x, y)\right), \quad x \in \Omega_{0}, \\
\chi\left(\Omega_{1 \varepsilon}^{h}\right) e\left(u_{\lambda}^{\varepsilon}(x)\right) \stackrel{2}{\rightarrow} \chi\left(Y^{h}\right)\left(e\left(u_{\lambda}(x)\right)+e_{y}\left(u_{\lambda}^{1}(x, y)\right)\right), \quad x \in \Omega_{1}, \\
\varepsilon \chi\left(\Omega_{\varepsilon}^{s}\right) e\left(u_{\lambda}^{\varepsilon}(x)\right) \stackrel{2}{\rightarrow} \chi\left(Y^{s}\right) e_{y}\left(w_{\lambda}(x, y)\right), \quad x \in \Omega_{1} .
\end{gathered}
$$

Under the above smoothness assumptions, (55)-(57) follow immediately from (61)-(63). This completes the proof of Theorem 1.

Finally, applying the inverse Laplace transform to (53)-(57), we deduce the following result.

Theorem 2. Let $u^{\varepsilon}(x, t)$ be a solution of problem (1)-(4). Then

$$
\begin{gathered}
\lim _{\varepsilon \rightarrow 0} \int_{\Omega_{\varepsilon}^{h}}\left|u^{\varepsilon}(x, t)-u(x, t)\right|^{2} d x=0, \quad \lim _{\varepsilon \rightarrow 0} \int_{\Omega_{\varepsilon}^{s}}\left|p^{\varepsilon}(x, t)-p(x, t)\right|^{2} d x=0, \\
\lim _{\varepsilon \rightarrow 0} \int_{\Omega_{\varepsilon}^{s}}\left|u^{\varepsilon}(x, t)-u(x, t)-w\left(x, \varepsilon^{-1} x, t\right)\right|^{2} d x=0, \\
\lim _{\varepsilon \rightarrow 0} \int_{\Omega_{0}}\left|e\left(u^{\varepsilon}(x, t)-u(x, t)-\varepsilon u^{0}\left(x, \varepsilon^{-1} x, t\right)\right)\right|^{2} d x=0, \\
\lim _{\varepsilon \rightarrow 0} \int_{\Omega_{1 \varepsilon}^{h}}\left|e\left(u^{\varepsilon}(x, t)-u(x, t)-\varepsilon u^{1}\left(x, \varepsilon^{-1} x, t\right)\right)\right|^{2}=0, \\
\lim _{\varepsilon \rightarrow 0} \int_{\Omega_{\varepsilon}^{s}} \varepsilon^{2}\left|e\left(u^{\varepsilon}(x, t)-w\left(x, \varepsilon^{-1} x, t\right)\right)\right|^{2} d x=0 .
\end{gathered}
$$

Here the pair $\{u(x, t), p(x, t)\}$ is the solution of the homogenized problem $(48)-(52), w(x, y, t)$ is given by (47), and

$$
u^{0}(x, y, t)=V^{k h}(y) \frac{\partial u_{k}}{\partial x_{h}}(x, t), \quad u^{1}(x, y, t)=-Q^{k h}(y) \frac{\partial u_{k}}{\partial x_{h}}(x, t)-p(x, t) Q(y),
$$

where the vector functions $V^{k h}(y), Q^{k h}(y)$, and $Q(y)$ are the solutions of the cell problems (27), (28), and (38), respectively. 


\section{References}

[1] G.Nguetseng, A general convergence result for a functional related to the theory of homogenization, SIAM J. Math. Anal., 20(1989), no. 3, 608-623.

[2] G.Allaire, Homogenization and two-scale convergence, SIAM J. Math. Anal., 23(1992), no. $6,1482-1518$.

[3] G.Allaire, M.Briane, Multiscale convergence and reitereted homogenization, Proc. of Royal Soc. Edinburgh, 126A(1996), 297-342.

[4] D.Lukassen, G.Nguetseng, P.Wall, Two-scale convergence, Intern. J. Pure and Appl. Math., $\mathbf{2 0}(2002)$, no. $1,35-86$.

[5] V.V.Zhikov, On an extention of the method of two-scale convergence and its applications, Mat. Sb., 191(2000), no. 7, 31-72 (in Russian).

[6] V.V.Zhikov, Homogenization of elasticity problems on singular structures, Izv. Ross. Akad. Nauk. Ser. Mat., 66(2002), no. 2, 81-148 (in Russian).

[7] G.Nguetseng, Asimptotic analysis for a stiff variational problem arising in mechanics, SIAM J. Math. Anal., 21(1990), no. 6, 1394-1414.

[8] R.P.Gilbert, A.Mikelić, Homogenizing the acoustic properties of the seabed: Part I, Nonlinear Analysis, 40(2000), no. 1, 185-212.

[9] Th.Clopeau, J.L.Ferrin, R.P.Gilbert, A.Mikelić, Homogenizing the acoustic properties of the seabed, Part II, Math. and Comput. Modelling, 33(2001), 821-841.

[10] G.W.Clark, R.E.Showalter, Two-scale convergence of a model for flow in a partially fissured medium, Electronic J. Diff. Equations, 2(1999), 1-20.

[11] A.Meirmanov, A description of seismic acoustic wave propagation in porous media via homogenization, SIAM J. Math. Anal., 40(2008), no. 3, 1272-1289.

[12] D.A.Kosmodem'yanskii, A.S.Shamaev, Spectral properties of some problems in mechanics of strongly inhomogeneous media, Izv. Ross. Akad. Nauk. Mekh. Tverd. Tela, 44(2009), no. 6, 75-114 (in Russian).

[13] A.S.Shamaev, V.V.Shumilova, Averaging the acoustic equations for a viscoelastic material with channels filled with a viscous compressible fluid, Izv. Ross. Akad. Nauk. Mekh. Zhidk. i Gaza, 46(2011), no. 2, 92-103 (in Russian).

[14] A.S.Shamaev, V.V.Shumilova, Homogenization of the acoustic equations for a porous long-memory viscoelastic material filled with a viscous fluid, Diffirentsialnye Uravneniya, 48(2012), no. 8, 1174-1186 (in Russian).

[15] E.Sanchez-Palencia, Non-Homogeneous Media and Vibration Theory, Lecture Notes in Physics, 127, Springer, 1980.

[16] Ya.I.Frenkel, On the theory of seismic and seismoelectrical phenomena in a moist soil, Izv. Acad. Nauk SSSR. Ser. Geogr. Geofiz., 8(1944), no 4, 133-150 (in Russian). 
[17] M.A.Biot, Mechanics of deformation and acoustic propagation in porous media, J. Applied Physics, 33 (1962), no. 4, 1482-1498.

[18] G.A.Chechkin, A.L.Piatnitski, A.S.Shamaev, Homogenization: Methods and Applications, Translations of Mathematical Monographs, 234, AMS, Providence, RI, 2007.

[19] O.A.Oleinik, A.S.Shamaev, G.A.Yosifian, Mathematical Problems in Elasticity and Homogenization, North-Holland, Amsterdam, 1992.

[20] L.R.Volevich, Solvability of boundary value problems for general elliptic systems, Mat. Sb., 68(1965), no. 3, 373-416 (in Russian).

\section{Усреднение уравнений акустики для частично перфорированного упругого материала со слабовязкой жидкостью}

\section{Алексей С. Шамаев Владлена В. Шумилова}

$\overline{\text { Рассмотрена математическая модель, описывающая малые колебания гетерогенной среды, состо- }}$ ящей из частично перфорированного упругого материала и слабовязкой сжимаемой жидкости, заполняющей поры. Для данной модели с помощью метода двухмасштабной сходимости построена соответствующая усредненная модель и найдены граничные условия, связывающие уравнения усредненной модели на границе между сплошным упругим материалом и пористым упругим материалом с жидкостъю.

Ключевые слова: усреднение, двухмасштабная сходимость, гетерогенная среда. 